\title{
Modeling Transit User Stop Choice Behavior: Do Travelers Strategize?
}

\author{
Mohammad Nurul Hassan, Taha Hossein Rashidi, and S. Travis Waller \\ University of New South Wales, Australia \\ Neema Nassir and Mark Hickman \\ University of Queensland, Australia
}

\begin{abstract}
Transit choice research focuses predominantly on mode choice and route choice, whereas very few studies on stop choice are conducted. To fill this gap, this research aimed to study transit stop choice behavior with a focus on how people strategize when making their choices. It is hypothesized that travelers treat stops differently based on various schemes (strategies); minimizing travel time, access time, and the number of transfers are the schemes considered in this study, and the effectiveness of several discrete choice model specifications was examined. The study found that path attributes and stop attributes have significant impacts on stop selection behavior. Furthermore, users' socioeconomic characteristics along with trip timing play important roles in choosing transit stops. The outcomes of this study could facilitate the recent move toward development of behavioral route choice models using smart card data, which can then assist travel demand estimation models with a focus on public transport.
\end{abstract}

Keywords: Transit stop choice, transit path choice, travel scheme, nested logit, mixed logit

\section{Introduction}

In transit demand modeling literature, two areas have been discussed: 1) transit mode choice (or general transit ridership) and 2) transit assignment or path choice. Recently, researchers have started using smart card data to develop transit path choice models (Schmöcker, Shimamoto, and Kurauchi 2013; Jánošíková, Slavík, and Koháni 2014). As smart card datasets can detect repetitive observations, path identification and estimation become much easier. By using a smart card dataset, Schmöcker, Shimamoto, and Kurauchi (2013) proposed a bi-level discrete choice model in which the upper level considers the choice preference of users and the lower level deals with the deterministic probabilities of boarding paths. However, as smart card datasets usually lack information 
about the actual origin and destination, these models can determine path choice from only the departure stop. Consequently, these models miss the link between the trip origin and departure transit stops.

This gap was addressed by Nassir et al. (2015) by developing a transit stop choice model. They assumed that transit users select their route by selecting a stop (bus stop, train station, or ferry terminal) from a desirable choice set. They argue that modeling the path choice behavior at the stop level is more appropriate, as the observed data are consistent with the choice actually made by the users. They proposed a nested structure in which an acceptable model fit is gained by considering a bi-level train and no-train nesting structure. Moreover, the study found that the choice of stop depends not only on the attributes of the paths (fastest travel time, number of transfers, etc.), but also on the attributes of the stops. They showed that the presence of shelter at stops, walk time from the origin location to the stop, travel time, number of transfers, and number of routes significantly affect the choice of stops. These findings add to the body of knowledge on the behavioral aspect of transit mode choice, but their work cannot be treated as a comprehensive stop choice study due to three major shortcomings: 1) they did not consider users' socioeconomic and demographic characteristics; 2) attributes related to the trip were missing; and 3) their modeling specification was quite limited and restricting.

Other stop choice studies are found in the literature, but they focused on other issues. Debrezion, Pels, and Rietveld (2009) conducted a railway station choice model for Dutch railway users. The main focus of their study was to determine a measure of station accessibility. They proposed a nested logit model in which access modes are modeled at the upper level and stations are modeled at the lower level. They found that access distance has a negative effect on the accessibility indicator, and parking availability, frequency of public transport, and railway station quality have a positive effect on station choice. Chakour and Eluru (2013) modeled access modes and station choice using a different approach. They found that a latent segmentation technique delivers better results than the nested logit approach proposed by Debrezion, Pels, and Rietveld (2009). Mahmoud, Habib, and Shalaby (2014) investigated the choice of parkand-ride stations for cross-regional commuter trips in the greater Toronto and Hamilton area. The study aimed to find aspects important to the design of more sustainable and attractive transit stations. They developed several multinomial logit models by using data on parking facilities, surrounding land use, and station amenities.

The work presented in this paper aimed to develop a stop choice model by addressing the shortcomings of the model developed by Nassir et al. (2015) and also to introduce a strategy-based (scheme-based) decision-making mechanism for transit users, which is a unique contribution from this paper. As such, we considered a total of 28 variables containing users' socioeconomic and demographic attributes and 9 variables addressing trip attributes, along with path attributes, stop attributes, and correction attributes. We also considered three strategy attributes. This study investigated appropriate modeling structures by testing different discrete choice models from the Household Travel Survey (HTS) of 2009 in Southeast Queensland (SEQ), Australia. The detailed description of 
the model is presented in the next section, followed by model results, discussions, and conclusions.

\section{Description of the Model}

In this study, it was assumed that when a transit user wants to make a trip, he/she decides what type of travel scheme is suitable for his/her current situation. In this study, we considered three basic schemes: minimize the time of travel (MTT scheme), minimize the access time (MAT scheme) to reach the boarding stop, and minimize the number of transfers (MTr scheme). Combinations of these three basic schemes (four combinations) also were considered. We assumed that users choose the alternative (access stop) that best matches their desired scheme and maximizes their utility. For example, if a user wants to minimize travel time (an MTT user), he/she chooses an alternative that falls under the MTT scheme. Similarly, an MAT-MTr user chooses a stop that takes less time to access and has the most direct connection to the destination (MAT-MTr scheme). The detailed descriptions of the models are discussed later in this section.

\section{Model Structure}

We considered four types of model structures: Multinomial Logit (MNL), Mixed MNL, Nested Logit (NL), and Mixed NL. In the MNL structure, the restricting Independence of Irrelevant Alternatives (IIA) property holds. This model forms the base case scenario. The form of MNL can be described by Equation (1):

$$
P_{n i}=\frac{e^{\beta^{\prime} x_{n i}}}{\sum_{i} e^{\beta^{\prime} x_{n i}}}
$$

where, $P_{n i}$ is the probability of selecting the alternative $i$ by an individual $n, x_{n i}$ is the column vector associated with attributes influencing the choice, and $\beta^{\prime}$ is the vector of parameters to be estimated.

A Mixed MNL model also was tested to determine if it could capture random taste variations among individuals. In the Mixed MNL formulation, $\beta^{\prime}$ is treated as a random parameter to be estimated, having a probability density function of $f(\beta)$. The choice probability of the Mixed MNL form can be written by the form provided in Equation (2). To capture the effects of the three basic schemes in MNL and Mixed MNL models, dummy variables (whether or not the option offers the scheme) were considered, because no nesting structure can be included in these models.

$$
P_{n i}=\int \frac{e^{\beta^{\prime} x_{n i}}}{\sum_{j} e^{\beta^{\prime} x_{n j}}} f(\beta) d \beta
$$

The third type, NL, was chosen to capture the correlation between alternatives belonging to different travel schemes. We assumed that alternatives falling under the same scheme have some unobserved similarities among them, and a nested structure might be able to capture them. Here, the schemes were considered to form the nests 
and the stops associated with the schemes were included under that nest. In the NL formulation, the choice probability for alternative $i \in B_{k}$ can be written as in Equation (3):

$$
P_{n i}=\frac{e^{\beta^{\prime} x_{n i} / \lambda_{k}}\left(\sum_{j \in B_{k}} e^{\beta^{\prime} x_{n j} / \lambda_{k}}\right)^{\lambda_{k}-1}}{\sum_{l=1}^{K}\left(\sum_{j \in B_{l}} e^{\beta^{\prime} x_{n j} / \lambda_{l}}\right)^{\lambda_{l}}}
$$

The fourth model, Mixed NL, can capture both random taste variations and correlation among the alternatives. Recently, some researchers (Hess, Bierlaire, and Polak 2005; Antonini, Bierlaire, and Weber 2004; Bajwa et al. 2008; Hammadou et al. 2008) reported a technique in which the $\beta^{\prime}$ coefficients inside the nests are treated as random parameters with a function of $f(\beta)$. The nest coefficients were not assumed to have any distribution. The model can be written as in Equation (4):

$$
P_{n i}=\int \frac{e^{\beta^{\prime} x_{n i} / \lambda_{k}}\left(\sum_{j \in B_{k}} e^{\beta^{\prime} x_{n j} / \lambda_{k}}\right)^{\lambda_{k}-1}}{\sum_{l=1}^{K}\left(\sum_{j \in B_{l}} e^{\beta^{\prime} x_{n j} / \lambda_{l}}\right)^{\lambda_{l}}} f(\beta) d \beta
$$

In the mixed models, randomness was captured assuming a log-normal distribution for the variables that show negative signs in MNL models, a uniform distribution for dummy variables, and a normal distribution for all the other variables (Hensher and Greene 2002).

Several studies focusing on the optimal choice of transit users combine all costs into a unified generalized cost to be considered in the objective function. Unlike this approach, this study attempted to introduce a "behavioral" stop selection model that reflects the process of decision-making by travelers. This behavioral model assumes that travelers maximize their utility based on the attributes of alternatives as well as a random error component capturing what is not known to the modeler. The proposed behavioral model is unique in a sense that it takes into account different ways to capture the unobserved error component in the utility function. It also examines mixed formulations to capture complicated taste variation structures.

\section{Nest Structures}

In this study, we considered three schemes (MTT, MAT, and MTr) individually and their combinations. Therefore, seven nesting groups were analyzed (see Table 1). These nesting structures also were used in Mixed NL estimation models. Thus, each group consisted of two models: NL and Mixed NL. The idea of considering different schemes as nests derived from the findings of other researchers (Nassir et al., 2015; Kurauchi et al., 2012; Fonzone and Bell 2010; Fonzone et al. 2010). Nassir et al. (2015) showed that transit users tend to choose stops that minimize travel time, minimize access time, and minimize the number of transfers. Kurauchi et al. (2012) found that London Oyster Card users might use different schemes (strategies) for their regular commute because they do not use fixed routes. Fonzone and Bell (2010) and Fonzone et al. (2010) also reported similar findings. 
TABLE 1.

Nest Structures for Proposed NL and Mixed NL Models

\begin{tabular}{|c|l|c|l|}
\hline Group & \multicolumn{1}{|c|}{ Model Name } & $\begin{array}{c}\text { Number } \\
\text { of Nests }\end{array}$ & \multicolumn{1}{|c|}{ Nest Structure } \\
\hline 1 & $\mathrm{TT}, \mathrm{TT}[\mathrm{M}]^{*}$ & 2 & MTT, NoMTT \\
\hline 2 & $\mathrm{AT}, \mathrm{AT}[\mathrm{M}]$ & 2 & MAT, NoMAT \\
\hline 3 & $\mathrm{Tr}, \operatorname{Tr}[\mathrm{M}]$ & 2 & MTr, NoMTr \\
\hline 4 & $\mathrm{TT}-\mathrm{AT}, \mathrm{TT}-\mathrm{AT}[\mathrm{M}]$ & 4 & MTT, MAT, MTT\&MAT, None \\
\hline 5 & TT-Tr, TT-Tr[M] & 4 & MTT, MTr, MTT\&MTr, None \\
\hline 6 & AT-Tr, AT-Tr[M] & 4 & MAT, MTr, MAT\&MTr, None \\
\hline 7 & TT-AT-Tr, TT-AT-Tr[M] & 8 & $\begin{array}{l}\text { MTT, MAT, MTr, MTT\&MAT, MTT\&MTr, } \\
\text { MAT\&MTr, MTT\&MAT\&MTr, None }\end{array}$ \\
\hline
\end{tabular}

* $[\mathrm{M}]=$ Mixed $N L$ model

In Table 1, the first nesting group is for the MTT scheme. Here, we considered two nests: 1) stops that are fastest (fastest routes from the stop) were grouped in the MTT nest, and 2) the rest of the stops were grouped in NoMTT nest. The next two groups considered the MAT and MTr schemes, similar to the first nesting group. The next three groups $(4,5$, and 6$)$ coupled two schemes; for example, in the fourth structure, both MTT and MAT were coupled. Here, there were four probable combinations of these two schemes: 1) minimizing travel time only (MTT), 2) minimizing access time only (MAT), 3) considering both (MTT and MAT), and 4) considering none of them (None). The last structure considered all three schemes, with all the probable combinations (eight nests).

\section{Data Preparation}

\section{Descriptive Analysis}

The dataset used in this research was taken from the Household Travel Survey (HTS) of May 2009 conducted in Southeast Queensland, Australia. All travel records (1,693 journeys) using public transport (which includes three modes: bus, train, and ferry), with walking legs of access, egress, and transfer(s), were extracted from the HTS data for this research. These 1,693 journeys included 1,435 transit trips with no transfers, 229 trips with a single transfer, 26 trips with 2 transfers, and 3 trips with 3 transfers. Regarding the mode of the access stop, 1,176 travelers had chosen bus stops, 492 travelers had chosen train stations, and 25 had chosen ferry terminals. The Queensland Department of Transport and Main Roads (DTMR) provided another dataset containing information about stop facilities such as shelter, lighting, access walkways, boarding slabs, etc. The SEQ transit authority Translink shared transit network data and service schedules for May 2009. The transit network included 14,442 stops, 767 paths, and 33,897 scheduled trips. The walk network data, consisting of local streets, sidewalks, crosswalk connections, walking ramps, footways, and stairways for SEQ, were obtained from OpenStreetMap (http://www.openstreetmap.org/). This included about 250,000 nodes and 340,000 links. ArcGIS was used to calculate the shortest walking paths. The average walking speed of a traveler was assumed to be $1.2 \mathrm{~m} / \mathrm{s}$ to calculate walking times. 
At the end of the choice set generation process, 1,238 observations were finalized. The scheme preferences of users for selecting their access stops were revealed from these data. A "reasonably minimum" travel time and access time were fixed for each choice set to account for the fact that users' perception of time does not exactly match reality. It was considered likely that an alternative stop yielding a travel time that was reasonably close to the minimum travel time of that choice set would be considered by an MTT user (who chooses a minimizing travel time scheme). To calculate the "reasonably minimum" travel/access time for a choice set, $10 \%$ of the difference between the maximum and minimum travel/access times was added to the minimum travel/access time. Stops that yielded less than this "reasonably minimum" travel/ access time threshold were flagged as MTT or MAT stops. For the MTr scheme, only the minimum number of transfers was considered. Finally, to be consistent with the relevant nesting group, separate data files were generated for each model. The revealed choice of schemes for each nesting group is presented in Figure 1.

FIGURE 1.

User preference of schemes

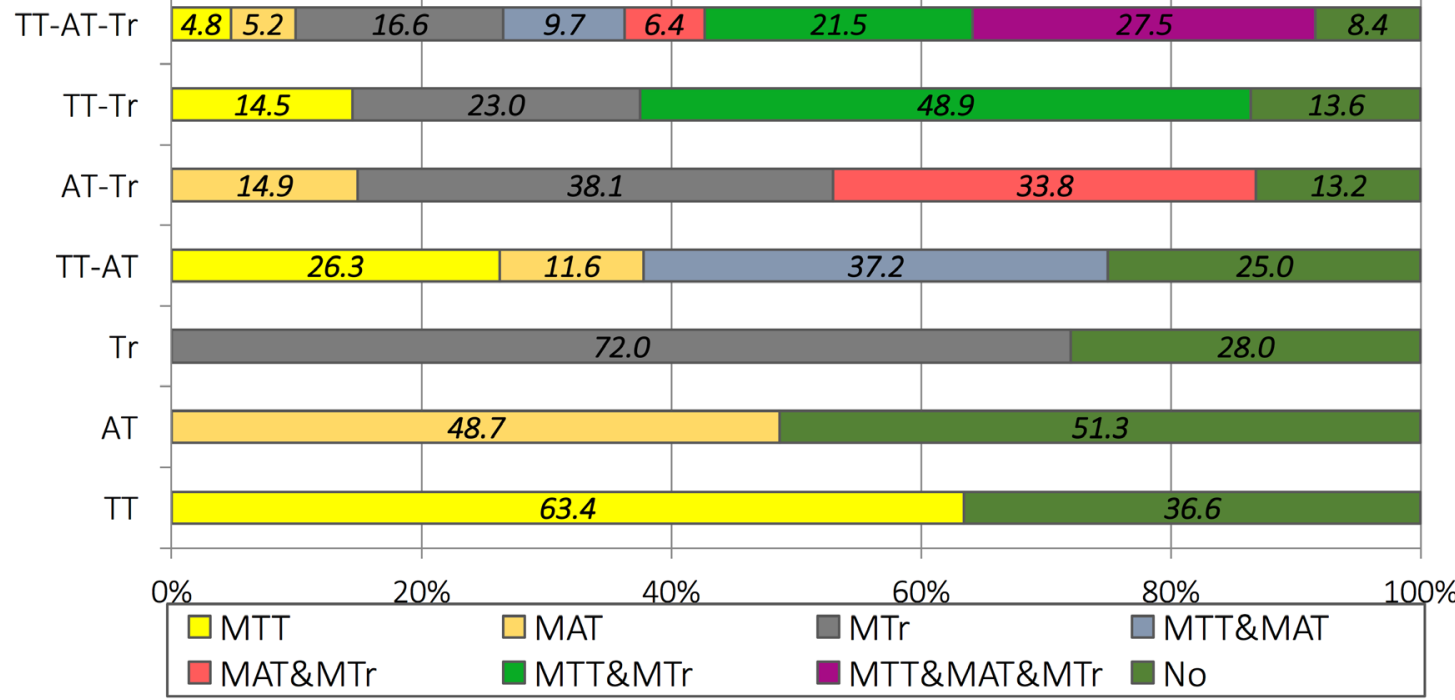

Figure 1 shows that most travelers choose access stops that contain some schemes. In the three-single-scheme situation, MTT and MTr schemes seem to be more popular (63\% of users choose MTT and 71\% choose MTr) than MAT schemes (only $49 \%$ of users choose MAT schemes). If there are multiple schemes, users seem to prefer combined schemes rather than single schemes or none. For example, in TT-AT and TT-Tr, the share of combined schemes are dominant (MTT\&MAT=37\%, MTT\&MTr=49\%) compared to single schemes or none. Contrastingly, in the AT-Tr combination, the share of $M T r$ (38\%) is more than the combined schemes of MAT\&MTr (34\%). Finally, in the TT-AT-Tr combination, users seem to prefer combined schemes. Very few (8\%) users seem to have no preference for schemes. 


\section{Explanatory Variables}

Several stop choice works were studied to develop the explanatory variables. Debrezion, Pels, and Rietveld (2009) mainly considered station facility attributes to construct their model. Chakour and Eluru (2013) considered socio-demographic attributes, trip characteristics, facility attributes, and land-use and built-environment factors. Mahmoud, Habib, and Shalaby (2014) studied facility attributes and land use variables. Nassir et al. (2015) considered facility attributes, impedance attributes, and correction attributes. In this study, we considered a total of 61 explanatory variables, which could be classified in 6 classes: 1) facility attributes, 2) impedance attributes, 3) user attributes, 4) trip attributes, 5) strategy attributes, and 6) correction attributes. Brief descriptions of the variables are provided in Table 2.

TABLE 2. Explanatory Variables of Models

\begin{tabular}{|c|c|c|c|}
\hline Variable & Mean & SD & Description \\
\hline \multicolumn{4}{|l|}{ Facility } \\
\hline AccessWalk & 11.33 & 7.11 & Walk time from origin location to stop (min) \\
\hline Shelter & 0.41 & 0.49 & Binary variable indicating sheltered stop \\
\hline StopLight & 0.34 & 0.47 & Binary variable indicating illuminated stop \\
\hline StreetLight & 0.31 & 0.46 & Binary variable indicating illuminated street \\
\hline BoardingSlab & 0.88 & 0.32 & Binary variable indicating existence of boarding slab \\
\hline FootPath & 0.87 & 0.34 & Binary variable indicating existence of foot path \\
\hline Map & 1.65 & 2.74 & Total number of printed map/schedule at stop \\
\hline \multicolumn{4}{|l|}{ Impedance } \\
\hline FastestTT & 46.95 & 19.85 & Travel time (min) of fastest path to destination from stop (excluding AccessWalk) \\
\hline MinTransfer & 0.83 & 0.84 & Minimum number of transfers among paths from stop to destination \\
\hline MinWalk & 19.05 & 8.88 & Minimum walk time (min) among paths from stop to destination (excluding AccessWalk) \\
\hline MinFare & 1.15 & 1.10 & Minimum fare among paths from stop to destination \\
\hline MinWait & 10.56 & 14.34 & Minimum wait time ( $\mathrm{min}$ ) among paths from stop to destination \\
\hline NumRoutes & 1.74 & 1.96 & Number of available paths from stop to destination \\
\hline TotalFreq & 4.55 & 7.46 & Summation of frequency for all paths from stop to destination \\
\hline AveTT & 48.50 & 20.33 & Average travel time of all paths from stop to destination (excluding AccessWalk) \\
\hline AveTransfer & 0.95 & 0.83 & Average number of required transfers for all paths from stop to destination \\
\hline AveWalk & 19.70 & 8.83 & Average walking time ( $\mathrm{min}$ ) for all paths from stop to destination (excluding AccessWalk) \\
\hline AveFare & 1.17 & 1.10 & Average fare for all paths from stop to destination \\
\hline AveWait & 12.37 & 15.17 & Average waiting time ( $\mathrm{min}$ ) for all paths from stop to destination \\
\hline \multicolumn{4}{|l|}{ User } \\
\hline Age & 35.55 & 19.20 & Age of user \\
\hline Male & 0.44 & 0.50 & Binary variable indicating user is male \\
\hline HHSize & 3.02 & 1.35 & Total number of members in $\mathrm{HH}$ (Household) \\
\hline CoupleKids & 0.36 & 0.48 & Binary variable indicating user $\mathrm{H} / \mathrm{H}$ type is couple with kids \\
\hline OneParent & 0.08 & 0.27 & Binary variable indicating user $\mathrm{H} / \mathrm{H}$ type is one parent with kids \\
\hline
\end{tabular}




\begin{tabular}{|c|c|c|c|}
\hline Variable & Mean & SD & Description \\
\hline Sole & 0.13 & 0.33 & Binary variable indicating user $\mathrm{H} / \mathrm{H}$ type is sole \\
\hline Couple & 0.20 & 0.40 & Binary variable indicating user $\mathrm{H} / \mathrm{H}$ type is couple \\
\hline OtherHHType & 0.23 & 0.42 & Binary variable indicating user $\mathrm{H} / \mathrm{H}$ type is other \\
\hline House & 0.81 & 0.40 & Binary variable indicating user lives in a house \\
\hline Flat & 0.15 & 0.35 & Binary variable indicating user lives in a flat \\
\hline Townhouse & 0.05 & 0.21 & Binary variable indicating user lives in a townhouse \\
\hline Bedrooms & 3.13 & 0.98 & Number of bedrooms in accommodation \\
\hline OwnedProp & 0.57 & 0.50 & Binary variable indicating user lives in owned property \\
\hline LivedInTheProp & 99.38 & 127.87 & Total number of months lived on accommodation \\
\hline HHIncome & $1,850.28$ & $1,340.09$ & Weekly income of $\mathrm{H} / \mathrm{H}$ \\
\hline HighPerIncome & 0.11 & 0.31 & Binary variable indicating user falls in high income group \\
\hline MedPerlncome & 0.38 & 0.49 & Binary variable indicating user falls in medium income group \\
\hline LowPerlncome & 0.51 & 0.50 & Binary variable indicating user falls in low income group \\
\hline FullTimeWork & 0.37 & 0.48 & Binary variable indicating user is full time worker \\
\hline AnyWork & 0.58 & 0.49 & Binary variable indicating user works \\
\hline Student & 0.00 & 0.06 & Binary variable indicating user is student \\
\hline AustralianBorn & 0.72 & 0.45 & Binary variable indicating user born in Australia \\
\hline CarLicence & 0.51 & 0.50 & Binary variable indicating user has car license \\
\hline BikeLicence & 0.02 & 0.15 & Binary variable indicating user has motorbike license \\
\hline NoLicence & 0.39 & 0.49 & Binary variable indicating user has no license \\
\hline TotalVehs & 1.37 & 0.99 & Total number of vehicles in $\mathrm{HH}$ \\
\hline PersonalVeh & 0.75 & 0.43 & Binary variable indicating user has personal vehicle \\
\hline Bicycles & 1.39 & 1.52 & Total number of bicycles in $\mathrm{HH}$ \\
\hline \multicolumn{4}{|l|}{ Trip } \\
\hline Train & 0.05 & 0.22 & Binary variable indicating trip access stop is train station \\
\hline AMPeakDep & 0.28 & 0.45 & Binary variable indicating trip starts in AM peak Hour \\
\hline PMPeakDep & 0.17 & 0.38 & Binary variable indicating trip starts in PM peak Hour \\
\hline PeakHourDep & 0.45 & 0.50 & Binary variable indicating trip starts in a peak Hour \\
\hline AMPeakArv & 0.25 & 0.44 & Binary variable indicating trip ends in AM peak Hour \\
\hline PMPeakArv & 0.22 & 0.42 & Binary variable indicating trip ends in PM peak Hour \\
\hline PeakHourArv & 0.48 & 0.50 & Binary variable indicating trip ends in a peak Hour \\
\hline Weekday & 0.90 & 0.30 & Binary variable indicating trip was on weekday \\
\hline PurposeWork & 0.67 & 0.47 & Binary variable indicating trip was made for work purpose \\
\hline \multicolumn{4}{|c|}{ Strategy (used only for MNL and Mixed MNL models) } \\
\hline MTTStr & 0.23 & 0.42 & Binary variable indicating option offers minimum travel time \\
\hline MTransferStr & 0.45 & 0.50 & Binary variable indicating option offers minimum number of transfers \\
\hline MAccessStr & 0.17 & 0.37 & Binary variable indicating option offers minimum walking access time \\
\hline \multicolumn{4}{|c|}{ Correction for Correlation } \\
\hline $\mathrm{CfC} 1$ & 1.09 & 0.79 & Correction for correlation, basic definition \\
\hline $\mathrm{CfC} 2$ & 1.09 & 0.79 & Correction for correlation, weighted by path frequency \\
\hline $\mathrm{CfC} 3$ & 1.09 & 0.79 & Correction for correlation, weighted by path travel time \\
\hline
\end{tabular}


Facility attributes included seven variables related to the transit stop. Two types of impedance attributes, direct and aggregate, were calculated from a path enumeration process. The path enumeration process refers to the procedure of generating a set of reasonable paths from a given origin and destination at the given departure time. Direct impedance attributes (the measures of best paths from different points of view) included five variables: fastest travel time, minimum number of transfers, minimum walking time, minimum fare, and minimum waiting time among all the reasonable paths from the origin to the destination. These, in fact, represented the best reasonable path in these five aspects from each stop. For example, for a particular stop, the fastest travel time variable indicated the fastest travel time of all reasonable paths from that stop. Similarly, the minimum number of transfers of all reasonable paths from the stop was recorded for the minimum transfer variable, and so on. Aggregate impedance attributes (including averages among all reasonable paths) included seven variables, among which five included the average measure (travel time, number of transfers, walking time, fare, and waiting time) among all reasonable paths. The other two contained the total number of possible paths from the access stop to destination and the total frequency of all these paths.

User attributes contained a variety of socio-economic attributes of the user. Trip attributes contained trip mode, timing, and trip purpose. Strategy attributes were used only for the MNL and Mixed MNL models. Corrections for correlation attributes were developed to deal with path commonalities (overlapping routes, which have strong correlations) among the stops. Path commonalities breach the IID (independent and identically-distributed) property of the MNL models to some extent and can lead to inaccurate estimations. The correction factors $(\mathrm{CfC} 1, \mathrm{CfC} 2, \mathrm{CfC} 3)$ proposed in this research were defined based on the Path Size Correction Logit (PSCL) formulation (Nassir et al. 2014). To meet the specifications of the access stop choice model, these factors were adjusted as follows (equations 5, 6, and 7). For an observation from origin location $o$ at departure time $\tau$ to destination location $d$, three definitions of correction for correlation were defined for every stop $s$ in the choice set $C_{o}^{d, \tau}$ :

$$
\begin{aligned}
& C f C 1_{s}^{d, \tau}=-\sum_{i \in \Gamma_{s}^{d, \tau} \tau} \frac{1}{\left|\Gamma_{s}^{d, \tau}\right|} \ln \sum_{t \in C_{o}^{d, \tau}} \delta_{i, t}^{d, \tau} \\
& C f C 2_{s}^{d, \tau}=-\sum_{i \in \Gamma_{s}^{d, \tau}} \frac{f_{i, s}^{\tau}}{\sum_{j \in I_{s}^{d, \tau} f_{j, s}^{\tau}} \ln \sum_{t \in C_{o}^{d, \tau}} \delta_{i, t}^{d, \tau}} \\
& C f C 3_{s}^{d, \tau}=-\sum_{i \in \Gamma_{s}^{d, \tau}} \frac{\left(T_{j, d}^{\tau}\right)^{-1}}{\sum_{j \in I_{s}, \tau}\left(T_{j, d}^{\tau}\right)^{-1}} \ln \sum_{t \in C_{o}^{d, \tau}} \delta_{i, t}^{d, \tau}
\end{aligned}
$$

Where $i, j$ are the indices of the routes; $s, t$ are the indices of stops; $\Gamma_{s}^{d, \tau}$ is the set of all routes at stop $\mathrm{s}$ with reasonable paths to destination $d$ at time $\tau ; f_{i, s}^{\tau}$ is the frequency of route $i$ at stop $s$ at time $\tau ; T_{j, d}^{\tau}$ is the travel time of the fastest path from stop s boarding on route $i$ to destination $d$ at time $\tau$; and $\delta_{i, t}^{d, \tau}$ is the top-route incidence parameter,

$\delta_{i, t}^{d, \tau}=\left\{\begin{array}{l}1, \text { if } i \in \Gamma_{s}^{d, \tau} \\ o, \text { if } i \notin \Gamma_{s}^{d, \tau}\end{array}\right.$ 


\section{Choice Set Generation}

Stop choice sets were generated by the algorithm in Nassir et al. (2015) in four steps (Figure 2). Initially, observed origin-destination and departure information (day and time) data were collected along with the walkway network and transit schedule. This information was used in the second step as an input. A version of a transit Trip-Based Shortest Path (TBSP) algorithm was used in this step (Khani et al. 2012; Nassir et al. 2012; Khani, Hickman, and Noh 2014; Khani 2013). This version is a transit time-dependent $\mathrm{K}$-shortest path algorithm that aims to minimize the arrival time to the destination and was modified to terminate after the destination was marked for computational efficiency.

FIGURE 2.

Choice set generation framework

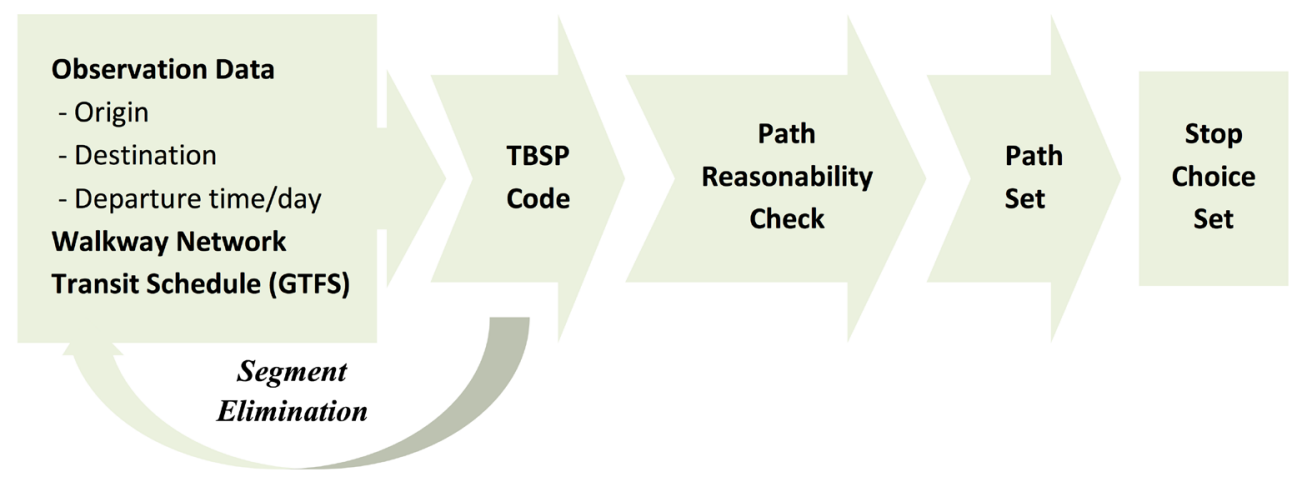

This algorithm has a "segment elimination" module that was executed after each iteration of the TBSP code. A segment is a combination of three elements: boarding stop, alighting stop, and the path connecting these two stops. In each iteration, after the TBSP generates a path, the segment elimination module eliminates all the segments used in that path from the schedule data and, thus, updates the schedule for the next iteration. This was done to create diversity among the generated paths.

In the third step, reasonable paths were sorted out. The TBSP code contained three reasonability conditions for path generation: 1 ) transfer walking distance cannot exceed $1 \mathrm{~km}, 2)$ access and egress walks cannot exceed $2 \mathrm{~km}$, and 3) waiting time before a boarding cannot exceed 1 hour. Two other reasonability checks also were set after the TBSP path generation: 1) path travel time does not exceed the shortest path travel time plus a threshold factor known as off-optimality, and 2) number of transfers does not exceed 3. The maximum off-optimality threshold was set as 20 minutes, as suggested in Nassir, Hickman, and Ma (2015).

The TBSP code also had an embedded maximum walking range of $2 \mathrm{~km}$ to generate the locations of the access stops from which the paths are generated. This $2 \mathrm{~km}$ threshold range was taken from the preliminary analysis of access walk from the SEQ HTS data, in which about $17 \%$ of the observations were found to walk more than $1 \mathrm{~km}$ to access to a transit stop (Nassir et al. 2015). At the end, the maximum number of stop choices in a set was found to be 70 stops, although the majority of observations had fewer than 20 stop choices in the set. Finally, based on the set of reasonable paths, impedance attributes and correction factors for each stop choice were calculated. 
It was found that the TBSP algorithm could select about $94.5 \%$ of the chosen access stops $(1,599$ out of 1,693$)$ successfully. The unsuccessful choices of stops were added in the choice sets manually. The impedance attributes of these stops were calculated by restricting the $\mathrm{K}$-shortest path generation algorithm to start from these stops. However, some observations were not matched to the exact stop location. We inferred these locations by applying three matching keys: whether the distance is within a $100 \mathrm{~m}$ threshold, the mode of the stops, and the path serving that stop. Ultimately, we had to exclude some of the observations (about 26.8\%), as we failed to locate the chosen access stop or observed ambiguity between the HTS data and generated paths.

\section{Model Results and Discussions}

The models were estimated using the discrete choice estimation package BIOGEME (Bierlaire 1998). Initially, all the models were estimated separately by one of the correction factors. Finally, the correction factors had to be dropped because these seemed to be insignificant, even at the $10 \%$ significance level. Table 3 provides a comparison between the models (MNL, NL, Mixed MNL, and Mixed NL) estimated in this study.

From Table 3, we can see that the MNL and NL models show similar adjusted $\rho^{2}$ values compared to the Mixed MNL and Mixed NL models. However, the Bayesian Information Criteria (BIC) values seem to be better in the MNL and NL models compared to the Mixed MNL and Mixed NL models. The model results indicate that two of the single-scheme NL models (AT model and TT model) result in significant nest structures. Nonetheless, in the single scheme Mixed NL models, the nest coefficients are insignificant. Furthermore, among the dual scheme models, TT-AT models show better nest structures and TT-Tr models show better BIC values than the other two groups. In contrast, most of the nest coefficients of the only tri-scheme model are insignificant, although their model fit (adjusted $\rho^{2}$ ) is better than all the other models. Therefore, from Table 3 we can conclude that travel schemes such as MTT and MAT have an influence on the users' choice of access stops; users generally follow MTT or MAT schemes or a combination of these two schemes (MTT-MAT).

From the comparisons shown in Table 3, we selected the best models according to three criteria: BIC, adjusted $\rho^{2}$, and significance of the nest coefficients. The MNL model shows the best BIC value among all the models; the adjusted $\rho^{2}$ value also is better than some of the models. The Mixed MNL model has a low BIC value compared to the MNL model, but the adjusted $\rho^{2}$ value is slightly better than the MNL model. Among the nested and mixed nested models, the TT-Tr and TT-Tr[M] models show the best BIC values (4012.04 and 4014.21, respectively). Moreover, the adjusted $\rho^{2}$ values also are higher than most of the other models in this group. Nevertheless, two of the nest coefficients of these two models seem to be insignificant (nest coefficient "None" was highly insignificant). On the other hand, TT and AT models have significant nest coefficients, but BIC and adjusted $\rho^{2}$ values seem to be worse than the other models in this group. However, if we want to balance the three criteria for model selection (BIC, adjusted $\rho^{2}$, and nesting coefficients), the TT-AT model can be considered as the best model among the nested and mixed nested models. The estimates of the MNL model and the TT-AT model are shown in Table 4. 
TABLE 3. Comparisons of Models*

\begin{tabular}{|c|c|c|c|c|c|c|c|c|}
\hline & \multirow{2}{*}{ MNL } & \multicolumn{7}{|c|}{ Nested Logit Models } \\
\hline & & TT & AT & $\operatorname{Tr}$ & TT-AT & TT-Tr & AT-Tr & TT-AT-Tr \\
\hline No. of parameters & 9 & 11 & 13 & 15 & 17 & 21 & 23 & 26 \\
\hline Final log-likelihood & -1970.858 & -1980.199 & -1985.941 & -1962.492 & -1951.951 & -1931.246 & -1943.24 & -1917.642 \\
\hline Likelihood ratio test & 1939.051 & 1915.459 & 1903.974 & 1950.872 & 1971.955 & 2013.364 & 1989.377 & 2040.369 \\
\hline$\rho^{2}$ & 0.329 & 0.326 & 0.324 & 0.332 & 0.336 & 0.343 & 0.339 & 0.347 \\
\hline Adjusted $\rho^{2}$ & 0.326 & 0.322 & 0.32 & 0.327 & 0.33 & 0.336 & 0.331 & 0.338 \\
\hline $\mathrm{BIC}$ & 4005.81 & 4038.73 & 4064.46 & 4031.80 & 4024.96 & 4012.04 & 4050.27 & 4020.44 \\
\hline \multirow[t]{3}{*}{ Nest Coefficients $(\lambda)$} & Not Applicable & $\begin{array}{l}\text { MTT }=0.81 \\
\text { NoMTT }=0.82\end{array}$ & $\begin{array}{l}\text { MAT }=0.78 \\
\text { NoMAT }=0.75\end{array}$ & $\begin{array}{l}M T r=0.79 \\
N o M T r=1.00 \\
(0.02,0.97)\end{array}$ & $\begin{array}{l}\text { MTT }=0.67 \\
M A T=0.81(1.32,0.19) \\
\text { MTT\&MAT }=0.63 \\
\text { None }=0.78\end{array}$ & $\begin{array}{l}\text { MTT }=0.83(1.43,0.15) \\
M T r=0.66 \\
\text { MTT\&MTr }=0.73 \\
\text { None }=0.99(0.13,0.9)\end{array}$ & $\begin{array}{l}\text { MAT }=0.90(0.78,0.44) \\
M T r=0.69 \\
M A T \& M T r=0.93(0.36,0.72) \\
\text { None }=0.96(0.52,0.61)\end{array}$ & $\begin{array}{l}M T T=0.71(1.35,0.18) \\
M A T=0.95(0.32,0.75) \\
M T r=0.63 \\
M T T \& M A T=0.68(1.21,0.23) \\
M T T \& M T r=1.00 \\
M A T \& M T r=0.38(1.03,0.3) \\
M T T \& M A T \& M T r=1 \\
\text { None }=0.98(0.31,0.76)\end{array}$ \\
\hline & \multirow{2}{*}{ Mixed MNL } & \multicolumn{7}{|c|}{ Mixed Nested Logit Models } \\
\hline & & TT[M] & $\mathrm{AT}[\mathrm{M}]$ & $\operatorname{Tr}[\mathrm{M}]$ & TT-AT[M] & $\operatorname{TT}-\operatorname{Tr}[\mathrm{M}]$ & $\mathrm{AT}-\operatorname{Tr}[\mathrm{M}]$ & $\operatorname{TT}-\mathrm{AT}-\operatorname{Tr}[\mathrm{M}]$ \\
\hline No. of parameters & 11 & 14 & 14 & 17 & 18 & 22 & 23 & 27 \\
\hline Final log-likelihood & -1966.73 & -1982.25 & -1974.53 & -1960.11 & -1976.32 & -1928.77 & -1938.57 & -1916.69 \\
\hline Likelihood ratio test & 1942.397 & 1905.092 & 1926.789 & 1955.643 & 1923.224 & 2018.315 & 1998.724 & 2042.266 \\
\hline$\rho^{2}$ & 0.331 & 0.325 & 0.328 & 0.333 & 0.327 & 0.343 & 0.34 & 0.348 \\
\hline Adjusted $\rho^{2}$ & 0.327 & 0.32 & 0.323 & 0.327 & 0.321 & 0.336 & 0.332 & 0.338 \\
\hline $\mathrm{BIC}$ & 4011.79 & 4064.19 & 4048.77 & 4041.28 & 4081.28 & 4014.21 & 4040.92 & 4025.66 \\
\hline Nest Coefficients $(\lambda)$ & Not Applicable & $\begin{array}{c}\mathrm{MTT}=0.89 \\
(1.2,0.23) \\
\text { NoMTT=0.91 } \\
(1.24,0.21)\end{array}$ & $\begin{array}{l}\text { MAT }=0.78 \\
(1.78,0.08) \\
\text { NoMAT }=0.76\end{array}$ & $\begin{array}{l}M T r=0.81 \\
\operatorname{NoMTr}=1 \\
(0.01,1.00)\end{array}$ & $\begin{array}{l}\text { MTT }=0.74 \\
\text { MAT }=0.79(1.44,0.15) \\
\text { MTT\&MAT }=0.63 \\
\text { None }=0.81\end{array}$ & $\begin{array}{l}\text { MTT }=0.85(1.23, \\
0.22) \\
M T r=0.65 \\
M T T \& M T r=0.75 \\
\text { None }=1(0.06,0.95)\end{array}$ & $\begin{array}{l}\text { MAT }=0.96(0.3,0.76) \\
\text { MTr }=0.66 \\
\text { MAT\&MTr }=0.93(0.33,0.74) \\
\text { None }=0.95(0.58,0.56)\end{array}$ & $\begin{array}{l}\text { MTT }=0.73(1.27,0.2) \\
M A T=1(.01,1.00) \\
M T r=0.63 \\
M T T \& M A T=0.74(0.97,0.33) \\
M T T \& M T r=0.54 \\
\text { AT Tr=0.3 }(0.97,0.33) \\
\text { MTT\&MAT\&MTr=1 } \\
\text { None }=0.94(0.8,0.42)\end{array}$ \\
\hline
\end{tabular}

${ }^{*}$ t-test value and $p$-value are provided in parentheses for coefficients that are not significant at $5 \%$ level. 
TABLE 4.

Estimation Results of Best Models

\begin{tabular}{|c|c|c|c|c|c|c|}
\hline \multirow[b]{2}{*}{$\begin{array}{c}\text { Explanatory Variables } \\
(\beta)\end{array}$} & \multicolumn{3}{|c|}{ MNL Model } & \multicolumn{3}{|c|}{ TT-AT Model } \\
\hline & Coefficient & $\begin{array}{l}\text { Robust } \\
\text { Std. Error }\end{array}$ & $\begin{array}{c}\text { Robust } \\
\text { t-test }\end{array}$ & Coefficient & $\begin{array}{l}\text { Robust } \\
\text { Std. Error }\end{array}$ & $\begin{array}{c}\text { Robust } \\
\text { t-test }\end{array}$ \\
\hline MinTransfer & -0.311 & 0.13 & -2.39 & -0.855 & 0.0772 & -11.07 \\
\hline MinWalk & -0.0329 & 0.0105 & -3.14 & -0.026 & 0.0091 & -2.85 \\
\hline NumRoutes & 0.0572 & 0.0141 & 4.07 & 0.048 & 0.0124 & 3.85 \\
\hline AccessWalk & -0.164 & 0.0133 & -12.39 & -0.124 & 0.0134 & -9.24 \\
\hline StopLight & 0.388 & 0.0951 & 4.08 & 0.292 & 0.0799 & 3.65 \\
\hline Train & 2.3 & 0.123 & 18.64 & 2.510 & 0.3110 & 8.06 \\
\hline MTTStr & 0.341 & 0.151 & 2.25 & \multicolumn{3}{|c|}{ N/A } \\
\hline MTransferStr & 1.030 & 0.176 & 5.83 & \multicolumn{3}{|c|}{$\mathrm{N} / \mathrm{A}$} \\
\hline AustralianBorn_TT & 0.435 & 0.177 & 2.46 & 0.825 & 0.1170 & 7.04 \\
\hline Male_TT_AT & \multicolumn{3}{|c|}{$\mathrm{N} / \mathrm{A}$} & -0.363 & 0.1620 & -2.25 \\
\hline Student_TT_AT & \multicolumn{3}{|c|}{$\mathrm{N} / \mathrm{A}$} & 9.730 & 0.7460 & 13.04 \\
\hline Flat_TT_AT & \multicolumn{3}{|c|}{$\mathrm{N} / \mathrm{A}$} & 0.569 & 0.1950 & 2.92 \\
\hline HHSize_TT_AT & \multicolumn{3}{|c|}{$\mathrm{N} / \mathrm{A}$} & 0.256 & 0.0343 & 7.47 \\
\hline PMPeakDep_TT_AT & \multicolumn{3}{|c|}{$\mathrm{N} / \mathrm{A}$} & -0.491 & 0.2390 & -2.05 \\
\hline \multicolumn{7}{|l|}{ Nest Coefficients $(\lambda)^{*}$} \\
\hline TT & \multicolumn{3}{|c|}{$\mathrm{N} / \mathrm{A}$} & 0.813 & 0.177 & $1.32+$ \\
\hline AT & \multicolumn{3}{|c|}{$\mathrm{N} / \mathrm{A}$} & 0.671 & 0.152 & 3.21 \\
\hline TT AT & \multicolumn{3}{|c|}{$\mathrm{N} / \mathrm{A}$} & 0.633 & 0.237 & 2.43 \\
\hline None & \multicolumn{3}{|c|}{$\mathrm{N} / \mathrm{A}$} & 0.781 & 0.079 & 3.58 \\
\hline
\end{tabular}

${ }^{*}$ Robust $t$-test is estimated for the hypothesis, $H_{0}=1$

+ Significant at 0.20 level

From Table 4, the two direct impedance attributes MinTransfers and MinWalk were found to be significant. The signs of these coefficients were negative, as expected; this means that transit users prefer to start their trip from a stop that had a more direct connection to their destination and involved less walking. One of the aggregate impedance attributes, NumofRoutes, was found to be significant in the models; this means that transit users tend to choose access stops that have multiple path options. Facility attributes AccessWalk and StopLight also were found to be significant. The negative sign of AccessWalk means users perceives more disutility if they have to walk more to the access stop. The positive sign of the StopLight attribute implies that users prefer to choose stops that have lighting. The sign of the coefficient of Train is positive, which means that transit users in SEQ are much more willing to travel by train than by other modes.

Generally, the coefficients of the common variables of these two models (presented in Table 4) seem to be quite similar, except for MinTransfer; the coefficient of MinTransfer was smaller in the MNL model. This probably happened because some of the effects of this parameter might have been captured by MTransferStr, which is a dummy variable for the presence of the MTr scheme. These models identify that users consider every minute of walking to the access stop to be about five minutes of other types of 
walking (e.g., for transfers or walking to the destination) involved in the travel path. This indicates that users do not perceive/evaluate walking in a consistent way. Somehow, walking to access stops poses a much higher disutility than other walks in the travel path. This might support theories about the myopic behavior of transit users by other researchers (Nassir et al. 2015; Fonzone and Bell 2010).

Compared to previous studies, in a nutshell, this study considered 61 attributes, compared to 21 attributes considered in Nassir et al. (2015). In analyzing the same dataset, the current study found 8 significant attributes in the MNL model and 12 significant attributes in the TT-AT model compared to 6 significant attributes in Nassir et al. (2015). Furthermore, the model fit (adjusted $\rho^{2}$ : MNL model 0.326 , TT-AT model 0.336 ) in this study seems to outperform the model fit (adjusted $\rho^{2}: 0.287$ ) developed by Nassir et al. (2015).

Some of the socio-economic attributes also were found to be significant. As reported in Table 4, both the models show that Australia-born users are more likely to select MTT schemes for choosing transit stops. In the TT-AT model, female students are more likely to use the combination of MTT and MAT schemes when choosing their preferred transit stop. Moreover, users from larger households and users living in a flat tend to prefer the combination of MTT and MAT schemes when choosing transit stops. Trip attribute PMPeakDep was found to be significant, indicating that users making a trip other than at the PM peak hour are inclined to follow the combined scheme of MTT and MAT when choosing their transit stop. Another interesting finding is that of the three strategy attributes used in the MNL model, two (MTT and MTr) became significant, which indicates that users consider either MTT scheme or MTr scheme. The NL model presented in Table 4 (TT-AT) shows significance ( $5 \%$ significance level) for the AT, TT-AT, and None schemes. The TT scheme is significant at the $20 \%$ level.

\section{Model Predictability and Sensitivity}

The choice probabilities of all the options were calculated for the MNL and TT-AT model. It was found that the models could correctly predict the users' chosen alternatives in 46\% (MNL) and 44\% (TT-AT) of cases. It also can be interpreted that, according to the MNL model, $46 \%$ of users choose the stop with the highest probability. Again, $84 \%$ of users (MNL model) seem to choose the access stop from a set of five stops with the highest probabilities; for TT-AT model, this is about $79 \%$. The predictive capabilities of these models are shown in Figure 3, which presents the cumulative percentage of successful prediction, with an increasing pattern for the number of options considered to include the actual selected option. In other words, if a set of predicted options is considered to include the observed option, the chance of having the observed option increases. Obviously, as the choice set (as defined previously in the methodology section) size increases, the chance of including the observed option in the set of predicted options decreases. In Figure 3, five curves are fitted, representing the prediction capabilities for having the observed choice in the set of predicted options where the highest probability is for curve 5 . This shows that the models can predict the 
choices better if the choice set size is relatively small, and vice versa. However, when the choice set size is larger than 40 , the predictability is uncertain.

FIGURE 3.

Prediction capabilities of stop choice models
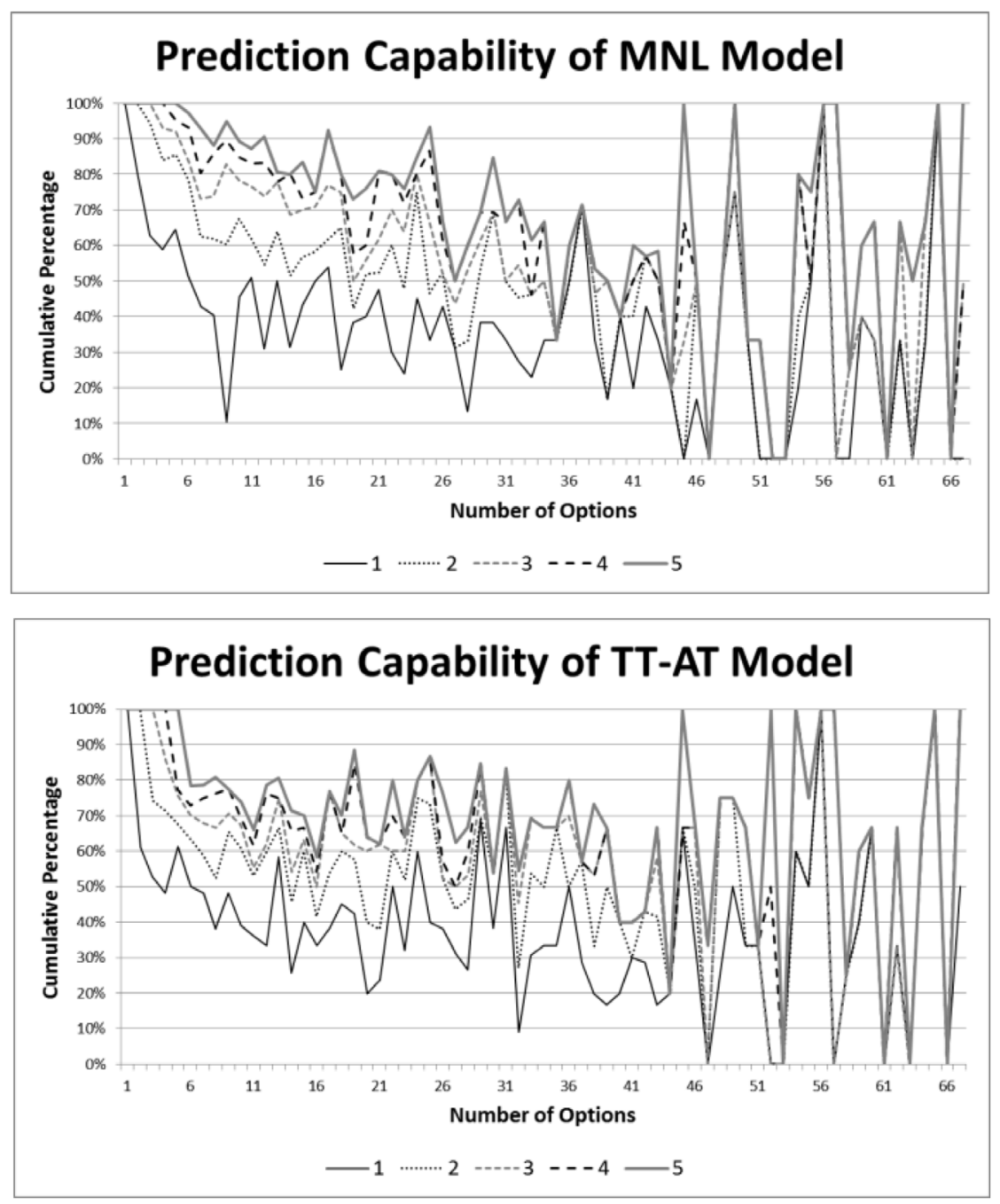

The TT-AT model was tested to observe the sensitivity of the nests with a change of access time and walking time; the results are shown in Figure 4. Here, the effects of waiting time seem to be almost similar to the effects of access time on the nests. However, the difference is in the magnitude, which seems to be much higher for the access time change. Figure 4 shows that by increasing the access time and waiting time, the probability of choosing from the AT and TT AT nests (strategies) increases; however, the TT nest seems to be relatively insensitive. This can be interpreted as follows: if the access time or waiting time is increased, the probability of selecting a stop that follows the MAT or MTT-MAT scheme will be increased, and if the access time or waiting time is decreased, the probability of selecting a stop that follows the MAT or MTT-MAT scheme will be decreased. 
FIGURE 4.

Effects of different variables

on nests
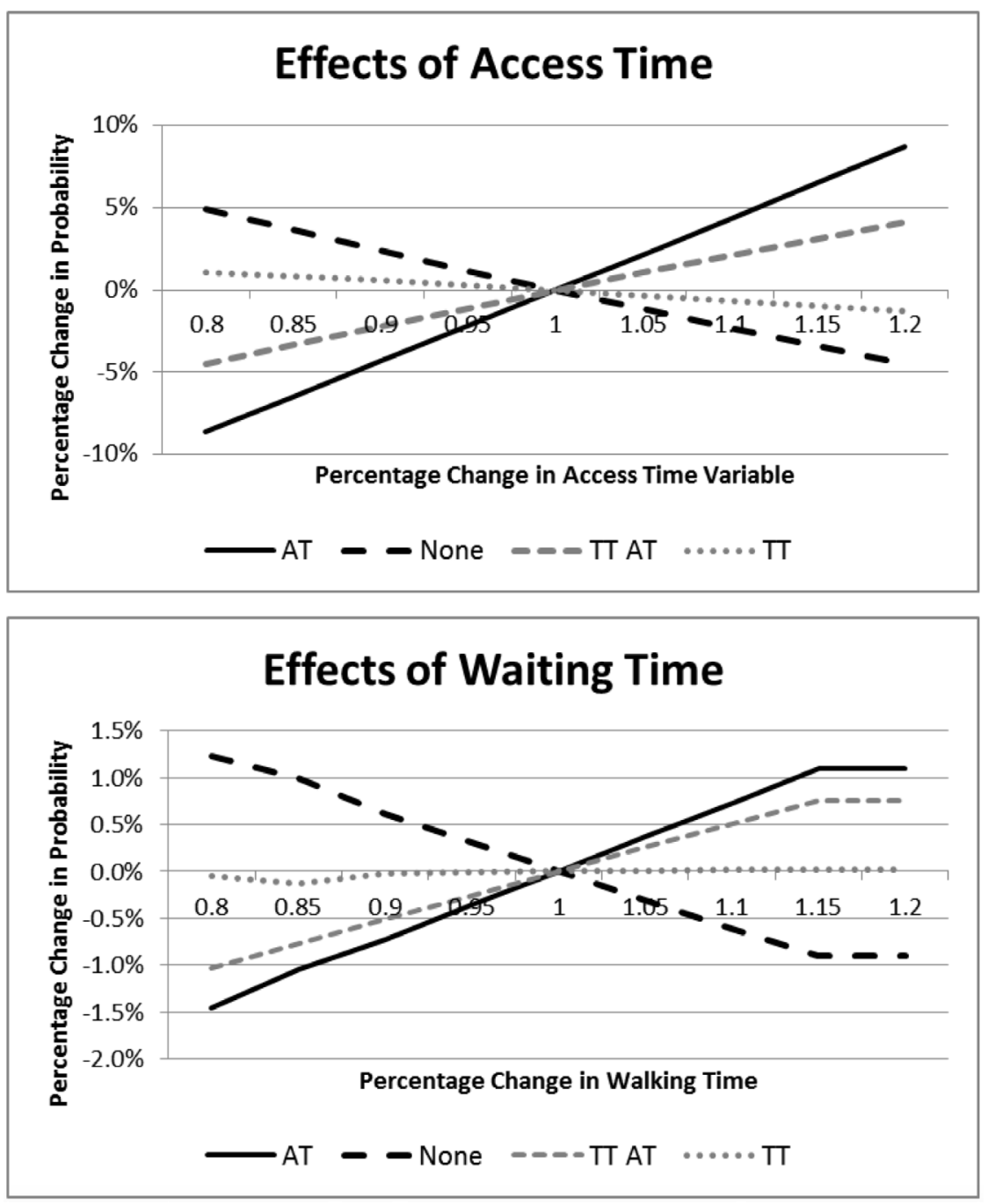

\section{Conclusions}

One of the contributions of this study is to improve the stop choice model developed by Nassir et al. (2015) by adding socioeconomic, trip, and strategy variables.

Furthermore, this study considered different nesting structures and developed several types of discrete choice models. Relating the nesting structures to the schemes/ strategies people consider when selecting stops is a unique contribution of this paper.

This study provides a deeper understanding about stop choice behavior compared to the existing literature. It was found that transit users can use different travel schemes/ strategies when selecting access stops. The most appropriate scheme seems to be the combination of minimizing travel time and minimizing access time. From the behavioral point of view, it can be concluded that SEQ transit users perceive alternatives that are either faster (MTT nest) or more easily accessible from the origin of the trip (MAT nest), or both (fast and nearby) in a similar way. 
This study shows that the choice of access stop is not only affected by impedance factors of the paths (number of transfers, walking time, travel time), but also by the attributes of the stop (such as walking time to access the stop and the presence of lighting at the stop). Moreover, the presence of multiple paths from a stop shows a positive influence on the utility of stop choices. Again, some socioeconomic attributes, such as gender, studentship, place of birth, household size, and dwelling type (flat), affect the choice of stop. Furthermore, transit users also take into account the transit mode and time of the day of the trip. One interesting point is that the developed models relate some of the impedance factors associated with paths linked to the origin and destination stops. Therefore, the proposed approach of this study married the stop and path selection themes in a straightforward manner, and further analysis is required to examine the opposite direction when stop attributes are includes in a route choice model. This work is underway by the authors.

The main contribution of this research is that it can be used to develop a behaviorbased transit path choice model from trip origin to destination. For this, the suggested access stop choice model can be developed from the trip origin to the departure stop. Again, from the departure stop to the destination stop, other boarding strategy-based models (from smart card data) can be developed. Eventually, the combination of these two models can effectively estimate and evaluate future transit demand from any given origin to destination. Thus, the presented study can be extremely beneficial for the policy-makers, as this eventually affects the evaluation process of transit policies considered for the target year.

Further investigations can be conducted to determine the impacts of travel schemes when paths are considered to be selected by travelers rather than stops. Other model structures, such as cross-nested logits, mixed cross-nested logits, and nested logits with multiple levels and combinations (e.g., scheme-mode-stop, scheme-mode-path, modescheme-path etc.), also can be tested. Results from such models can provide a clearer understanding about transit choice research.

\section{References}

Antonini, G., M. Bierlaire, and M. Weber. 2004. "Simulation of Pedestrian Behaviour Using a Discrete Choice Model Calibrated on Actual Motion Data." 4th Swiss Transport Research Conference.

Bajwa, Shamas, Shlomo Bekhor, Masao Kuwahara, and Edward Chung. 2008. "Discrete Choice Modeling of Combined Mode and Departure Time." Transportmetrica, 4(2): 155-77.

Bierlaire, Michel. 1998. "BIOGEME: A Free Package for the Estimation of Discrete Choice Models."

Chakour, Vincent, and Naveen Eluru. 2013. "Analyzing Commuter Train User Behavior: A Decision Framework for Access Mode and Station Choice." Transportation, 41(1): $211-28$. 
Debrezion, Ghebreegziabiher, Eric Pels, and Piet Rietveld. 2009. "Modelling the Joint Access Mode and Railway Station Choice." Transportation Research Part E: Logistics and Transportation Review, 45(1): 270-83. doi:10.1016/j.tre.2008.07.001.

Fonzone, Achille, and Michael G. H. Bell. 2010. "Bounded Rationality in Hyperpath Assignment: The Locally Rational Traveller Model." Paper presented at the 89th Annual TRB Meeting, Washington, DC. http://researchrepository.napier.ac.uk/4238/.

Hammadou, Hakim, Isabelle Thomas, Ann Verhetsel, and Frank Witlox. 2008. "How to Incorporate the Spatial Dimension in Destination Choice Models: The Case of Antwerp." Transportation Planning and Technology, 31(2): 153-81. doi:10.1080/03081060801948126.

Hensher, D. A., and William H. Greene. 2002. "The Mixed Logit Model : The State of Practice and Warnings for the Unwary." Working Paper, Institute of Transport Studies, Australia, 01-02. Institute of Transport Studies, the University of Sydney and Monash University.

Hess, Stephane, Michel Bierlaire, and John W. Polak. 2005. "Mixed GEV Modelling of Mode-Choice." Monte Verità/Ascona.

Jánošíková, L’udmila, Jiři Slavík, and Michal Koháni. 2014. "Estimation of a Route Choice Model for Urban Public Transport Using Smart Card Data." Transportation Planning and Technology, 37(7): 638-48. doi:10.1080/03081060.2014.935570.

Khani, Alireza. 2013. "Models and Solution Algorithms for Transit and Intermodal Passenger Assignment (Development of FAST-TrIPs Model)." University of Arizona.

Khani, Alireza, Mark Hickman, and Hyunsoo Noh. 2014. "Trip-Based Path Algorithms Using the Transit Network Hierarchy." Networks and Spatial Economics, 15(3): 63553.

Khani, Alireza, Sanggu Lee, Mark Hickman, Hyunsoo Noh, and Neema Nassir. 2012. "Intermodal Path Algorithm for Time-Dependent Auto Network and Scheduled Transit Service." Transportation Research Record, 2284: 40-46.

Kurauchi, Fumitaka, Jan-Dirk Schmöcker, Achille Fonzone, Seham Mohamed Hassan Hemdan, Hiroshi Shimamoto, and Michael G. H. Bell. 2012. "Estimating Weights of Times and Transfers for Hyperpath Travelers." Transportation Research Record, 2284 (-1): 89-99. doi:10.3141/2284-11.

Mahmoud, Mohamed, Khandker Habib, and Amer Shalaby. 2014. "Park-and-Ride Access Station Choice Model for Cross-Regional Commuting: Case Study of Greater Toronto and Hamilton Area, Canada." Transportation Research Record, 2419: 92-100. doi:10.3141/2419-09.

Nassir, Neema, Mark Hickman, Ali Malekzadeh, and Elnaz Irannezhad. 2015. "Modeling Transit Access Stop Choices." http://trid.trb.org/view.aspx?id=1338262.

Nassir, Neema, Mark Hickman, and Zhen-Liang Ma. 2015. "Activity Detection and Transfer Identification for Public Transit Fare Card Data." Transportation, 42(4): 683705. 
Nassir, Neema, Alireza Khani, Mark Hickman, and Hyunsoo Noh. 2012. "Algorithm for Intermodal Optimal Multidestination Tour with Dynamic Travel Times." Transportation Research Record, 2283: 57-66. doi:10.3141/2283-06.

Nassir, Neema, Jennifer Ziebarth, Elizabeth Sall, and Lisa Zorn. 2014. "Choice Set Generation Algorithm Suitable for Measuring Route Choice Accessibility." Transportation Research Record, 2430 (December): 170-81. doi:10.3141/2430-18.

Schmöcker, Jan-Dirk, Hiroshi Shimamoto, and Fumitaka Kurauchi. 2013. "Generation and Calibration of Transit Hyperpaths." Procedia-Social and Behavioral Sciences, 80: 211-30.

\section{About the Authors}

Mohammad Nurul Hassan (m.n.hassan@student.unsw.edu.au) is a Ph.D. candidate at the University of New South Wales (UNSW) in Australia.

TAHA Hossein RASHIDI (Rashidi@unsw.edu.au) is a Senior Lecturer at UNSW.

S. Travis WALLeR (s.waller@unsw.edu.au) is a Professor and rCITI Director at UNSW. Neema Nassir (n.nassir@uq.edu.au) is a Postdoctoral Research Fellow at the University of Queensland in Australia.

MARK HICKMAN (m.hickman1@uq.edu.au) is a Professor and ASTRA Chair of Transport at the University of Queensland in Australia. 